\title{
INFORMES
}

\section{ASPECTOS DEMOGRÁFICOS DEL DESARROLLO ECONÓMICO *}

\section{CONSIDERACIONES GENERALES}

Desde la publicación, en 1953, de Factores Determinantes y Consecuencias de las Tendencias Demográficas ${ }^{1}$ se ha registrado un aumento importante de la investigación sobre las interrelaciones entre los factores económicos y demográficos. ${ }^{2}$ Un examen sistemático de las últimas publicaciones realizado por la División de Población, que cubre el tiempo transcurrido desde 1953, indica que han aumentado tanto el alcance como la profundidad de las investigaciones sobre este tema. Parece que la población se considera cada vez más como un factor que influye en la determinación de las polí. ticas nacionales de desarrollo y de la planificación económica. También se han registrado cambios importantes en las actitudes de los grupos que deciden las políticas de las Naciones Unidas y de los organismos especializados interesados en los problemas demográficos y en el progreso económico y social.

A medida que ha aumentado el conocimiento de las relaciones económico-demográficas, se han hecho más evidentes las lagunas y deficiencias. Por lo tanto, los órganos que formulan las políticas de las Naciones Unidas han continuado insistiendo sobre la necesidad de una mayor investigación y de la adopción de medidas en la esfera económico-demográfica, especialmente en aquellos aspectos de interés inmediato y directo para los países en desarrollo.

Si el crecimiento de la población forma parte de los problemas y procesos del desarrollo económico en todo el mundo, el crecimiento rápido crea considerables dificultades para el desarrollo en la mayoría de los países que hoy están económicamente menos avanzados. Debido a estos cambios demográficos, casi todas las características de su escena económica, política y social han cambiado, están cambiando y continuarán cambiando.

Las tasas más altas de crecimiento de la población significan que el número de niños que han de ser educados, de bocas que se han de alimentar y de manos que se han de emplear sube más rápidamente de lo previsto. Recientemente, el rápido crecimiento de la población de los países en desarrollo ha aumentado la presión sobre los recursos agrícolas, de los que aún depende la mayoría de la gente, y también ha contribuido a las migraciones masivas internas, especialmente de las zonas rurales a las urbanas. En el sector urbano moderno de muchos países, la demanda de mano de obra ha crecido más lentamente que la fuerza de trabajo urbana alimentada por la migración; y los pobres en las ciudades, sea que estén desempleados o empleados con salarios muy bajos, son cada vez más.

* Extractado del informe del Comité Especial de Expertos en Programas sobre los Aspectos Demográficos del Desarrollo Económico, de las Naciones Unidas, reunido del 29 de junio al 3 de julio de 1970. Naciones Unidas, Consejo Económico y Social, Comisión de Población, Doc. E/CN.9/239, 15 de octubre de 1970.

1 Doc. ST/SOA/SER.A/28, Naciones Unidas, Departamento de Asuntos Económicos y Sociales, Nueva York, 1953, $77 \mathrm{pp}$.

2 Véanse las Actas de la Conferencia Mundial de Población, volúmenes I a IV (Publicación de las Naciones Unidas. Núm. de Venta: 66. XIII. 5 a 8). 
Las consecuencias cada vez más difundidas de este fenómeno reciente todavía no se comprenden bien, pero, a pesar del conocimiento limitado de la forma precisa en que el rápido crecimiento de la población cambia el funcionamiento y la estructura de una sociedad y de una economía, unos treinta paises han evaluado sus propias circunstancias y han comenzado a adoptar medidas para alentar una reducción de la fecundidad y para disminuir el crecimiento de la población. En la mayoría de los casos, estos acontecimientos han sido provocados, entre otras cosas, por los indeseables efectos económicos y sociales del rápido crecimiento de la población.

E1 propósito del Comité era identificar los aspectos que se necesita investigar con urgencia para comprender mejor las interrelaciones entre el crecimiento y el desarrollo de la economía y estas tendencias demográficas sin precedentes. Si bien se comprenden básicamente los mecanismos que relacionan los movimientos demográficos con el ritmo y carácter del desarrollo, todavía queda mucho por hacer en esta esfera. El mecanismo inverso, que relaciona los cambios en el medio económico y social con los niveles de mortalidad y especialmente de fecundidad que sostienen estas tendencias demográficas ha sido apenas explorado. Una política efectiva dependerá en último término del progreso en el conocimiento de las causas determinantes de la fecundidad, que es la variable crítica del rápido crecimiento de la población $\mathrm{y}$, por ello, constituye la segunda categoría de prioridad en la investigación.

\section{LA POBLACTÓN COMO PRODUCTORA Y CONSUMIDORA}

En el examen de los programas para el estudio de los aspectos demográficos del desarrollo económico, a menudo se ha subrayado el doble papel de la población como un conjunto de productores y de consumidores; el hombre es el productor básico de todos los bienes materiales y también su consumidor, tanto en lo que se refiere a las necesidades básicas de alimento, vestuario y vivienda como respecto de los servicios complementarios de sanidad, educación y cultura. Los cambios demográficos influyen primordialmente mediante la demanda del consumidor y la oferta de mano de obra sobre otros aspectos económicos y sociales de la sociedad.

El papel de "productora" y de "consumidora" de la población se considera tradicionalmente en términos de los grupos de edad del "ciclo vital", que comienza con los párvulos (0-4), cuya participación en la población total es especialmente importante en lo relativo a la demanda de alimentos, vivienda, y servicios médicos y de bienestar. La población en edad escolar (5-14) es considerada tradicionalmente como exclusivamente consumidora, pero en ciertas circunstancias también puede ser productora, invirtiendo asimismo parte de su tiempo y energías en el estudio. A medida que los niños egresan de la escuela e ingresan a la fuerza de trabajo, obtienen también, en la mayoría de las sociedades, la independencia económica necesaria como para pensar en el matrimonio. La población en edad de trabajar (generalmente 15-64 años) incluye tanto a las personas que prestan sus servicios dentro de la familia como a aquellas que laboran en lo que tradicionalmente se llama la fuerza de trabajo. Este grupo de productores es también el principal consumidor de bienes y servicios. El tamaño de este grupo económicamente activo en relación con el de la población que no trabaja, y depende de otros, es la llamada relación de dependencia. Éste es también el grupo de edad donde tienen lugar el matrimonio y la formación de una familia e incluye el importante grupo de los que están en la edad de reproducción (15-49 años). Después de haber proporcionado su labor productiva durante la mayor parte de su vida, la generación pasa a ser en forma definitiva económicamente inactiva. Este grupo vuelve a ser 
principalmente consumidor, aunque sus demandas pueden ser ahora menores que durante sus años de trabajo.

Este método demográfico tradicional para vincular el consumo económico y los aspectos productivos de la composición de la población se ha usado relativamente poco para esclarecer las condiciones dentro de los países o para formular comparaciones internacionales. Aunque en algunos países la falta de estadísticas adecuadas presenta un obstáculo serio para trabajar en estas direcciones, el análisis de las investigaciones sobre la familia puede proporcionar conocimientos sobre la naturaleza de las interrelaciones económico-demográficas que funcionan de manera diferente para los grupos de edad en diferentes etapas del ciclo vital.

\section{INTERACCIONES ECONÓMICAS Y DEMOGRÁFICAS}

El crecimiento de la fuerza de trabajo y su productividad, el ritmo y la estructura de la formación de capital y la utilización y el desarrollo de los recursos naturales constituyen el conjunto de fuerzas que figura en la base del estudio interdisciplinario de los aspectos demográficos del desarrollo económico. Este conjunto de factores incluye las relaciones más ocultas entre los factores demográficos y el ahorro, la inversión, el cambio y la eficiencia de la tecnología, y la fuerza de trabajo con sus realizaciones educativas y técnicas.

Sin embargo, el proceso de desarrollo es mucho más que un proceso homogéneo de incremento de factores. El desarrollo se entiende mejor como un proceso que produce una creciente participación de la población en actividades claramente diversas y más productivas. Estas actividades económicas requieren típicamente más servicios de capital y otros insumos modernos por trabajador que los usuales en otros sectores de la economía; y, como son menos rutinarios, requieren típicamente de la fuerza de trabajo más instrucción y conocimientos para hacer frente a métodos de producción cada vez más complejos y cambiantes. La tasa a que la fuerza de trabajo se absorbe en este sector moderno de la economía es restringida y el proceso de desarrollo generalmente se prolonga por el rápido crecimiento de la población, pues muchos empleos nuevos requieren que una mayor proporción del producto se ahorre e invierta y que se adquieran nue. vas especializaciones. En este modelo de cambio estructural en una economía "dual", las restricciones al desarrollo aumentan con el rápido crecimiento demográfico.

La población y la formación de capital. La discusión de los aspectos demográficos del ahorro se centra principalmente sobre la distribución por edades y las tasas de dependencia, que son en gran medida una función de la fecundidad y la mortalidad en cuanto afectan el suministro de ahorros originados en las familias. Se cree que los cambios en el crecimiento de la población y las consecuentes modificaciones en la distribución por edades afectan la capacidad de ahorro de dos maneras diferentes. La reducción de las necesidades de consumo, con un ingreso dado, que se asocian con una composición de edad menor, puede tender a aumentar el potencial de ahorro. Sin embargo, y lo que es más importante, los cambios en la capa. cidad productiva provenientes de una menor proporción de trabajadores en relación con los no trabajadores probablemente disminuirá el potencial de ahorro. Como se ha demostrado en cierto número de análisis y modelos a nivel macroeconómico, el alto grado de dependencia en los países en desarrollo ha hecho.más difícil la tarea ya formidable de generar ahorros suficientes para la formación de capital físico y el rápido crecimiento eco. nómico. 
Investigaciones recientes sugieren que debe darse mayor importancia a los efectos de los factores demográficos sobre el ahorro a nivel del hogar o de la familia. Los efectos del tamaño y la composición de la familia sobre el ingreso, el ahorro y el consumo han sido estudiados, pero principalmente en los países de altos ingresos. El modelo hipotético y más complicado de ahorro basado en el ciclo vital, en el cual la distribución por edades de los productores y consumidores se presenta como un determinante potencial del ahorro, puede constituir un marco aún mejor para estudiar en los países de bajos ingresos los efectos de las condiciones demográficas cambiantes a la altura de la familia.

Sin embargo, también se ha advertido que los aumentos en la capacidad potencial de ahorro resultantes de cambios demográficos tales como la disminución de la fecundidad no aumentan necesariamente la corriente de ahorros familiares. En los países en desarrollo, con su característicamente alta propensión al consumo, debida a los bajos niveles del ingreso y a su desigual distribución, un potencial de ahorro más alto posibilitado por una menor fecundidad, podría ser orientado hacia un aumento del consumo en lugar de ser ahorrado. Se necesitan investigaciones que aíslen los efectos de los cambios demográficos sobre el consumo, el ahorro y la inversión en las diversas condiciones sociales y económicas que prevalecen en los países en desarrollo.

El rápido crecimiento de la población en los países en desarrollo no sólo tiende a disminuir la oferta de ahorros disponible para inversiones, sino que también implica la necesidad de un alto nivel de inversión para mantener o aumentar los niveles de vida. El argumento es, por supuesto, que en los países en desarrollo, donde se cree que el capital es el factor críticamente escaso de la producción, el crecimiento del producto es restringida por la inversión. El efecto del crecimiento de la población sobre las necesidades de formación de capital se ilustra con mucha frecuencia por medio del modelo Harrod-Domar, reconocido como demasiado simplificado. Dada la relación marginal capital-producto, la proporción del ingreso que debe invertirse para mantener por lo menos los niveles de vida prevalecientes es igual a la relación marginal capital-producto - cuya magnitud total se estima a menudo en aproximadamente 3 - multiplicada por la tasa de crecimiento de la población. Expuesto en otra forma, dado el volumen de inversión y la relación marginal capital-producto, mientras más alta sea la tasa de crecimiento de la población, mayor es la parte de las llamadas inversiones demográficas que deben dedicarse a la absorción del efecto del crecimiento de la población, y menor es la parte disponible para aumentar los niveles de ingresos.

El Comité advirtió las deficiencias de este enfoque y de otros similares, pero no negó el importante papel de la formación de capital en el desarrollo económico y los efectos probables de los factores demográficos sobre el nivel y la distribución de la inversión. Se señaló que las tendencias demográficas propenderían a afectar la inversión en capital físico en el sector privado solamente en la medida en que, como factores demográficos, influyeran sobre el nivel y la composición de la demanda de diferentes bienes y servicios. Más bien se atribuyó importancia a las repercusiones del crecimiento de la población sobre los niveles y distribución de las inversiones en el sector público, el cual, con su estrecha base tributaria, soporta la mayor parte de los costos de las inversiones asociadas con una población joven, que se está urbanizando y crece rápidamente. Además, la distribución de las inversiones entre inversiones directamente productivas e inversiones sociales se traslada hacia la demanda ya muy considerable de inversiones sociales en escuelas, viviendas, hospitales y otras similares. Aunque la inversión en los sectores sociales es esencial, sus beneficios económicos aparecen a largo plazo y el efecto inmediato es canalizar los recur- 
sos para inversión fuera de los usos y sectores directamente productivos.

La población y el empleo. La interacción entre la población y la economía probablemente no es en ninguna parte tan directa como en el caso del empleo y el desempleo. Uno de los problemas más urgentes que afrontan los países en desarrollo es, en la opinión del Comité, la utilización apropiada de sus recursos humanos, caado el rápido crecimiento de su fuerza de trabajo, resultante de las altas tasas de crecimiento de la población en la posguerra.

Los altos niveles de desempleo y subempleo se consideraron fundamentalmente como resultado de que la demanda de mano de obra no absorbe la oferta creciente. Sin embargo, la inflexibilidad e inelasticidad de la oferta de mano de obra también se mencionaron como posibles factores de la menos que óptima utilización de los recursos de trabajo disponibles, del desempleo de temporada y de la persistencia de las pautas de trabajo tradicionales que se han citado.

Se opinó que los factores institucionales, tales como la rigidez de los sistemas de tenencia de Ia tierra para la agricultura, son una causa importante de la relativa lentitud de la expansión del empleo en muchos países. Fuera de estos obstáculos institucionales, la escasez de medios cooperativos de producción fue uno de los factores principales en la explicación del des. empleo y del subempleo en los países menos desarrollados. El rápido crecimiento de la población agrícola, en relación con los recursos de tierra disponibles, produce o aumenta el subempleo o el abierto desempleo. Análogamente, los niveles de inversión en muchos países en desarrollo son demasiado bajos para absorber a la fuerza de trabajo en rápido crecimiento. El crecimiento económico en términos de un indicador como el "ingreso" no contribuye necesariamente en forma significativa a solucionar el problema del empleo si las técnicas de producción están orientadas en favor de los métodos basados en grandes inversiones de capital. La escasez de ciertos tipos de trabajadores especializados, principalmente para la industrialización, o la falta de capacidad empresarial, también pueden ejercer una influencia desfavorable sobre el crecimiento del empleo en general.

Se convino en que la demanda de mano de obra aumentó en las últimas décadas como resultado del crecimiento económico, pero en general se estimó que este crecimiento no se mantuvo a la par con el de la oferta. En opinión de los participantes, las investigaciones y estudios en la última década han sugerido un creciente desequilibrio entre la oferta de mano de obra y la demanda, creando niveles más altos de desempleo abierto y una incidencia mayor de subempleo. En opinión del Comité, uno de los problemas más importantes de los países en desarrollo es el del desempleo y el subempleo, y la repercusión de las tendencias demográficas en esta esfera requiere más profundas investigaciones cuantitativas para elaborar las políticas apropiadas.

Aspectos demográficos del progreso tecnológico y del cambio institucional. El proceso de la correcta aplicación de una tecnología más eficiente está asociado a un conjunto de factores que a su vez lo condicionan; estos factores incluyen la capacidad empresarial, los cambios institucionales, el desarrollo de las asociaciones humanas, el mejoramiento tanto del capital físico como humano, etc. Entre las interacciones entre la población y los factores económicos, las más complicadas son aquellas entre el tamaño, la composición y las tendencias de la población, por una parte, y el cambio tecnológico, por la otra.

En ciertas épocas, el rápido crecimiento de la población puede haber ayudado al progreso tecnológico, como en el caso de los países industriali. 
zados de hoy durante la revolución industrial y después. Los adelantos tecnológicos del transporte y de las comunicaciones aceleraron la migración al extranjero. La ampliación de los mercados puede haber estimulado otras innovaciones tecnológicas, aunque el valor de las economías de escala nacionales nunca ha sido establecido plenamente.

El proceso de crecimiento económico en los países en desarrollo de hoy es diferente en muchos aspectos. Su intento de saltar etapas del proceso, por ejemplo mediante la importación de tecnología con gran densidad de capital, se complica por el hecho de que la tasa de crecimiento de la población es mucho más rápida que lo que fue la de los países desarrollados en períodos anteriores. La transferencia de la tecnología moderna que ahorra mano de obra a las regiones menos desarrolladas, que tienen abundante mano de obra no calificada, origina problemas muy conocidos de formación de capital, empleo y productividad, cuyos aspectos han sido bien estudiados en lo que se llama economía dual. Las prácticas mercantiles discriminatorias de los países industrializados, que protegen sus industrias y segmentos de la agricultura menos eficientes, limitan severamente las ganancias que los países en desarrollo pueden obtener hoy del comercio internacional.

El Comité advirtió que las posibilidades de desarrollar y de introducir el progreso tecnológico, adaptado a las condiciones de los países en desarrollo, como ha ocurrido en el caso de la agricultura y la ganadería, deberían estudiarse mejor. También discutió la posibilidad de que, al promover tecnologías con menos densidad de capital, los efectos de creación de empleos podrían compensar el mayor costo que según se presume acarrea el uso de estos métodos. La adopción lisa y llana por los países en desarrollo de la tecnología desarrollada en los países avanzados, bajo la influencia de muy diferentes precios relativos de los factores, no parece justificada por razones de eficiencia ni de equidad. Las repercusiones demográficas de la tecnología con gran densidad de capital en condiciones de rápido crecimiento de la población no se comprenden adecuadamente. Se señaló que, al evaluar la capacidad de la tecnología con gran densidad de capital para absorber mano de obra, debía tenerse en cuenta la creación indirecta de empleos en las industrias conexas y especialmente en el sector moderno de servicios. En cambio, se formuló por ejemplo la interrogante de si la presión de una población rural en expansión no podría conducir a una adaptación tecnológica adecuada mediante cambios en la composición de las cosechas o en las técnicas de cultivo o ambas cosas a la vez. La gravedad del problema fue reconocida, especialmente respecto de la población marginal de las zonas urbanas.

Aspectos demográficos de la transformación estructural. Debido a la novedad del marco socioeconómico y a las características distintivas de la presente transición demográfica en los países en desarrollo, las teorías demográficas formuladas para explicar los cambios históricos observados en los países avanzados pueden proporcionar sólo poca orientación para el futuro.

Sin embargo, la prueba limitada de la experiencia histórica en los países industrializados sugiere que los elementos más avanzados o modernos de la sociedad tienden a reducir su fecundidad primero, y que la ampliación gradual del desarrollo tiende a acelerar la aceptación de la familia pequeña como norma, hasta que este proceso reduce la fecundidad nacional. Si este esquema es válido también para los países en desarrollo de hoy, el cambio global en el crecimiento de la población se convierte en un proceso integral de interacción entre fecundidad, mortalidad y migración por una parte, y cambios en la estructura socioeconómica de la población, especialmente debidos a la industrialización, por la otra. 
El proceso de desarrollo económico no se lleva a cabo exactamente en la misma forma en todos los países. Hay muchas diferencias notables, especialmente en la forma como se produjo en los países avanzados y como ahora se está produciendo en los países en desarrollo. Sin embargo, ciertos cambios estructurales básicos de naturaleza demográfica son características comunes de los procesos de desarrollo en todos los países. Varios miem. bros del Comité subrayaron que el cambio de las actividades agrícolas a las no agrícolas y la migración de las zonas de residencia rurales a las urbanas es acompañado por un importante mejoramiento en la "calidad" de la población. Una fuerza de trabajo gradualmente mejor instruida, el sensible mejoramiento de la condición jurídica y social de la mujer y, en muchos casos también, los cambios estructurales en la organización de la familia con una creciente centralización de la responsabilidad en la familia nuclear, son condiciones necesarias para la utilización efectiva de la nueva tecnología y para la organización más racional de los proceđimientos de trabajo.

Estos cambios estructurales en la población y en la economía se produjeron como parte integrante de la historia del desarrollo en los países avanzados. Es evidente que su efecto combinado fue el de modificar las pautas de reproducción, aumentando el costo de la crianza de los niños y ofreciendo al mismo tiempo a los padres opciones alternativas y competitivas tanto para el uso de su tiempo libre como de sus recursos financieros. Generalmente no ha sido posible determinar el punto de partida de una determinada transformación estructural en la que comienza a producirse un cambio en la fecundidad o siquiera identificar cuál de los cambios estructurales involucrados es el más importante para el cambio en la fecundidad.

En realidad, es más probable que la relativa importancia de los diferentes cambios de que se trata varie de un país a otro siguiendo las diferentes formas que haya adoptado el proceso de desarrollo y el contexto histórico en que ocurre el proceso. Por ejemplo, hay razones para creer que la condición mejorada de la mujer fue un factor más importante de este proceso de cambio en la Unión Soviética y en los países socialistas de Europa Oriental debido al gran interés que estos países tenían en el cambio del papel tradicional de la mujer y el aumento de su participación en la fuerza de trabajo.

Estos cambios se reflejan no solamente en las mayores oportunidades abiertas a la mujer para realizar una variedad mayor de actividades econó. micas fuera del hogar, sino también en la elevación de su nivel cultural y de su condición juridica y social.

Se requiere con urgencia que se produzcan transformaciones estructurales de esta clase en los países en desarrollo. Varios miembros del Comité instaron a que se prestara atención a determinados aspectos de los progra. mas de desarrollo y a su relación con las transformaciones estructurales que probablemente afecten a la población y a su conducta reproductiva. Por ejemplo, aunque en ciertos casos puede ser más económico ubicar a las nuevas industrias en zonas rurales en lugar de llevar a la población a las ciudades, los cambios estructurales involucrados serian menores y tendrían poco efecto sobre las normas de vida. Análogamente, la mayor actividad económica de la mujer puede tener muy poco efecto sobre la fecundidad si ocurre en circunstancias que se combinan fácilmente con Ia crianza de los niños, tal como en las artesanías de aldea. Las políticas de desarrollo tienen su influencia en el cambio demográfico y deberían examinarse a fondo para lograr que concuerden con los objetivos económicos y demográficos nacionales.

La densidad de la población. El Comité no pudo ponerse de acuerdo sobre el papel de la densidad de la población para evaluar las probables 
consecuencias sociales y económicas de la alta fecundidad y del rápido crecimiento de la población. La mayoría del Comité opinó que el rápido crecimiento de la población debido a la alta fecundidad imponía una carga sustancial sobre los miembros de la sociedad y sobre el gobierno que trataba de educar, alojar y proporcionar atención médica al creciente número de menores. Sin embargo, es útil distinguir entre dos grupos básicos de países en desarrollo con alta fecundidad y rápido crecimiento de la población: los de alta y los de baja densidad de población. La densidad, a juicio del Comité, no debía medirse simplemente como población por unidad de superficie de tierra, o quizás de tierra arable, sino que debia ser un concepto más significativo que se relacionara con la cantidad y la distribución de los recursos naturales y de capital disponibles por persona en una zona. Se sostuvo que la baja densidad se asociaba con una situación en que la población era pequeña en relación con las oportunidades económicas, la infraestructura y los recursos de una región. Las redistribuciones regionales de población se justificarían en tales casos por motivos puramente económicos, aunque el crecimiento más rápido de la población total no se justificaba por motivos únicamente económicos.

En las regiones de baja densidad con suficiente superficie de buena tierra para agricultura, el crecimiento de la población agrícola a veces puede no conducir a la forma común de superpoblación agrícola. En efecto, el aumento en la densidad de la población puede inducir en tales casos una organización agrícola más productiva con el desarrollo simultáneo de un transporte eficiente y el suministro de insumos modernos para la agricultura, tales como abonos, semillas y servicios mecanizados. Se informó al Comité que al presente, en una parte de Brasil -la región costera, poblada con relativa densidad- una gran proporción de la población está concentrada en 15 a 20 grandes aglomeraciones urbanas muy dispersas. Estas zonas están conectadas por una red de transporte y comunicaciones que, sin embargo, es cara y antieconómica porque la población que vive en las zonas intermedias está esparcida y en disminución. En casos como éste, la infraestructura social y no una mayor población puede ser una mejor solución para el problema del desarrollo económico. Pero el Comité convino en que las relaciones económico-demográficas subyacentes en la dinámica de la política del desarrollo regional merecen mayor estudio detallado tanto en los países avanzados como en los en desarrollo.

\section{ANÁLISIS ECONÓMICO DEL CRECIMIENTO DE LA POBLACIÓN}

El análisis económico puede aplicarse productivamente al estudio de las poblaciones y los problemas sociales que se relacionan con diferentes tasas y estructuras de crecimiento de la población. Aunque se necesita urgentemente una teoría integrada y un método empírico de estudio de la demografía económica, el estado actual de los conocimientos no está suficientemente unificado ni es bastante amplio como para deducir con confianza las causas precisas de varios acontecimientos demográficos o sus consecuencias sobre los objetivos económicos y sociales de un país. El Comité discutió dos criterios generales para el estudio de los determinantes sociales y económicos y las consecuencias de las tendencias de la población: 1) macrométodos que buscan estudiar la manera en que ocurren las interacciones entre la población, su estructura y su composición, por una parte, y la producción agregada y el proceso global de transformación estructural en la economía, por otra, y 2) micrométodos que tratan de descubrir cómo surgen los cambios demográficos, dados Ios factores sociales, culturales y económicos que actúan fuera de la familia, del comportamiento 
familiar y la forma en que estas normas de conducta alteran las oportunidades subsiguientes de los miembros de la familia por el resto de sus días, lo que contribuye a su vez al proceso general de desarrollo social y económico. La integración de estos dos niveles de teoría y análisis económico y demográfico es uno de los mayores desafíos para la futura investigación en este campo.

Macromodelos. Si bien el Comité reconoció que los macromodelos demográfico-económicos han contribuido a una mejor comprensión de ciertos aspectos de las correlaciones demográficas y económicas en la década pasada, opinó no obstante que la mayoría de estos modelos siguen siendo menos que satisfactorios como guía para quienes establecen las políticas, porque no toman en cuenta la complejidad esencial de estas correlaciones y no se apoyan todavía en forma segura sobre relaciones de conducta em. píricamente verificadas. De acuerdo con esta clase general de modelo, una reducción en la tasa de natalidad reduce la proporción de la población que es demasiado joven para participar en la fuerza de trabajo. Por consiguiente, si la producción o el ingreso agregados no son afectados por la tasa reducida de natalidad durante una década o más, el ingreso por habitante debe elevarse en comparación con el caso de las tasas constantes de natalidad. Aún más, si se supone que las tasas de ahorro aumentan en respuesta a este aumento relativo del ingreso per capita se acumulan nuevas ventajas económicas a largo plazo para la población que experimenta la tasa de natalidad declinante. Este método da por sentado que un ingreso (o consumo) creciente por habitante es un adecuado objetivo social, y que funciones simples de producción acumulativa (típicamente con dos factores, mano de obra y capital) describen adecuadamente las perspectivas de crecimiento a largo plazo de los países en desarrollo. Se han agregado ciertos supuestos adicionales en algunos modelos para tomar en cuenta la necesidad de realizar inversiones sociales que se relacionan con la composición por edades, el efecto de la calidad de la fuerza de trabajo, y los rendimientos decrecientes que se experimentan frente al suministro limitado de tierra y recursos naturales.

Aunque estos macromodelos demográfico-económicos constituyen un adelanto sobre las formas más sencillas del modelo Harrod-Domar, donde el crecimiento de la producción es sólo función de la inversión y la contribución del trabajo se ignora a veces implícitamente, estos macromodelos sin embargo descuidan esferas importantes de interacción y retroacción que pueden ser más significativos que las repercusiones directas del cambio en las tasas de fecundidad y de mortalidad. Como se subrayó anteriormente, la relación entre las declinaciones de la fecundidad y los aumentos en los ahorros y las inversiones familiares potenciales merece un estudio empírico. Algunos miembros del Comité opinaron que mientras los modelos macroeconómicos tradicionales ponen énfasis hasta cierto punto justificable en la formación de capital físico, pueden haber descuidado las fuentes no tradicionales de crecimiento económico que deben explicar el crecimiento secular de la productividad del trabajo y del capital. Los determinantes de esta fuente residual de crecimiento pueden estar estrechamente ligados a un grado más alto de educación en la fuerza de trabajo, a un mejor estado de salud y nutrición en la fuerza de trabajo, y a la mejor distribución regional y sectorial del trabajo y el capital.

Al considerar las deficiencias de los macromodelos existentes de correlaciones económico-demográficas, el Comité indicó varias direcciones en que podria encaminarse la investigación para revelar herramientas más realistas y poderosas para el trazado de políticas y la planificación de largo alcance. Es necesario incorporar a estos modelos más detalles de la estruc. 
tura de la población y no sólo de su tamaño y tasa de crecimiento. Por ejemplo, se asocia a la educación con diferencias en la productividad de la mano de obra y también se la liga frecuentemente con diferenciales de fecundidad. La migración intersectorial de la mano de obra responde a la industrialización, llevando gente de las zonas rurales a las urbanas con probables repercusiones sobre la productividad y la fecundidad de la mano de obra. Investigaciones más amplias del cambio demográfico y económico intentarían incorporar algunos de estos mecanismos mediante los cuales el desarrollo económico y social altera la distribución regional de la población, cambia su composición industrial, educacional y ocupacional, y quizás influencie su conducta procreadora. Esta cadena de relaciones específicas necesita una mayor investigación empírica antes de que pueda ser incorporada en forma útil a los macromodelos económico-demográficos.

Micromodelos. Se presta creciente atención a los análisis y las investigaciones que interpretan el proceso del cambio demográfico y económico desde el punto de vista de la unidad familiar. Este método puede tener especial pertinencia para la comprensión de los determinantes de la fecundidad y las consecuencias de la declinación de la fecundidad, aunque se señaló que estos modelos se han usado principalmente en los países más desarrollados y que sin modificaciones no serían necesariamente válidos en los países en desarrollo. Analíticamente, este método, que integra los fenómenos económicos y demográficos, supone que los individuos ejercen un grado de elección en lo concerniente a su participación en la fuerza de trabajo, el estado civil, el comportamiento migracional y, en especial, la fecundidad. Después de averiguar qué factores podrían influir generalmente a la gente en cada una de estas esferas de conducta, este método analítico aplica análisis con múltiples variables para calcular empíricamente el sig. nificado estadístico y la magnitud de estas influencias postuladas sobre el comportamiento. A la inversa, los aspectos económicos del comportamiento tales como la participación en la fuerza de trabajo o la modalidad de ahorro se interpretan ambas como función de las oportunidades y restricciones económicas y las características demográficas anteriores del individuo y de su unidad familiar. Como medio para interpretar las pautas de procreación, este método no debería ser estrechamente económico, sino que debe comprender a las instituciones sociales, el estado de las partes productivas, el bienestar social y económico y los recursos de la familia.

La información acumulada de esta manera sobre los determinantes locales de varias esferas de conducta económica y demográfica debe proporcionar gradualmente una base científica para seleccionar de entre varias políticas de desarrollo aquellas que alienten los cambios de población que sean consecuentes con el avance social y económico más rápido de la sociedad. Este método promete también clarificar las consecuencias de varias tendencias demográficas, al documentar los cambios en los niveles de consumo y las oportunidades sociales que surgen de diferentes tipos de conducta demográfica.

Los estudios de familias y de pequeñas comunidades que experimentan las presiones del rápido crecimiento de población deben revelar cuáles son las consecuencias del número y la edad de los niños sobre el consumo de la familia y las pautas de ahorro, como también sobre la participación en la fuerza de trabajo admitiendo un ingreso familiar y determinantes ambientales del tamaño familiar deseado. Estos estudios deberían evaluar las ventajas comparativas de tener más niños y de mantener las oportunidades de consumo de que disfrutan los miembros existentes de la familia, tales como mejor alimentación, servicios de sanidad, educación y mayores oportunidades de migración. Con este método es posible calcular cómo los regí- 
menes alternativos de fecundidad y planificación de la familia afectan los ahorros familiares físicos y la conducta de inversiones en el ciclo vital.

Construcción de un puente entre los micro y macromodelos. Las rela. ciones de conducta estimadas sobre la base de la observación de familias durante el cambio económico y demográfico pueden proporcionar el fundamento para cla rificar los supuestos arbitrarios que generalmente sirven de base a los modelos macroeconómicos de este proceso. Por ejemplo, la elección de funciones de ahorro puede calcularse para cada país y clase donde el ingreso o la fecundidad estén variando. En el futuro se puede usar la decreciente mortalidad infantil, los ingresos en aumento y la creciente asistencia escolar para calcular con mayor precisión con qué rapidez probablemente cambien las pautas de fecundidad por edades. Es posible que tanto teórica como empíricamente los microanálisis proporcionen la base para un mayor realismo en la formulación de macromodelos para la planificación a largo plazo y la evaluación de políticas en la esfera demográfica y económica.

Asimismo, el Comité también puso de relieve que la sociedad y la economía no son simplemente la suma de las unidades familiares sino que hay interacciones externas entre las familias y otros agentes económicos. Las firmas particulares y el sector público también constituyen tema de análisis y modelos económicos, aunque el sector familiar merece mayor inves tigación. El paso más difícil para integrar un micro-macromodelo de des. arrollo económico-demográfico será la elaboración de los medios para tratar los costos sociales (externos a la familia) de la alta fecundidad que pesan sobre la sociedad a través de efectos difusos sobre el mercado privado, tales como un mayor desempleo, salarios más bajos, menor movilidad social o económica y que crean también obligaciones sociales fuera de la familia con sus demandas de mayores servicios del sector público para sanidad, enseñanza y posiblemente viviendas construidas con fondos públicos. El cálculo de estas interacciones entre las familias y el sector público es una difícil tarea de investigación que aún no ha sido intentada en los países industrializados o en aquellos en desarrollo.

\section{Métodos para EValuar las opciones entre las polf́ticas}

Análisis de costo/beneficio. Las discusiones anteriores sobre los costos y beneficios de los proyectos de población se concentraron en la microevaluación de un nacimiento "marginal". En un análisis reciente llevado a cabo por el Banco Internacional de Reconstrucción y Fomento hubo un vuelco hacia un método macroeconómico basado en modelos demográficoeconómicos. Este estudio intenta medir los efectos de los gastos que tienden a influir la tasa de crecimiento de la población mediante el cálculo: a) del tamaño de la población futura, y $b$ ) del ingreso nacional futuro. Si un objetivo básico lo constituye un creciente ingreso nacional por habitante, es posible calcular los efectos de los gastos sobre este objetivo.

Se arguyó que el método macroeconómico ofrece ventajas tanto teóricas como prácticas sobre la metodología alternativa. Los costos y beneficios de un nacimiento marginal no han sido siempre persuasivos; los valores relativamente altos de los beneficios en relación a los costos, junto con las dificultades para manejar algunos problemas conceptuales, significaron que los resultados obtenidos fueran a menudo recibidos con escepticismo. E1 método macroeconómico da también tasas muy altas de rendimiento, o bajos promedios de costo/beneficio, pero éstos pueden considerarse en relación con el impacto general de los gastos propuestos en la economía.

Hay cierto número de problemas que merecen discusión y estudio más difundidos; algunos de ellos son asuntos de concepto, teoría y disponibili- 
dad de datos, tales como el papel del descuento, la tasa de descuento que se va a usar y la elección de una función objetiva. Una pregunta clave es cuál función de ahorros se va a usar dada la escasez de información acerca de la conducta relativa al ahorro en los países en desarrollo. Igualmente crucial es la elección de la función de producción que se usa en cualquiera de esos análisis macroeconómicos.

Al usar el análisis de costo/beneficio tales cálculos deben ser solamente una parte del proceso de tomar decisiones respecto de gastos. La metodología se aplica generalmente a la asignación de recursos en el sector público donde no es posible o no es deseable confiar en el mecanismo de asignación del mercado. Se encuentran en este dominio los gastos relacionados con los programas de planificación de la familia subvencionados con fondos públicos, como también otros programas de gastos públicos que influyen con medios voluntarios en el tamaño y la tasa de crecimiento de la población. Sin embargo, el carácter social de estos programas crea dificultades para la correcta evaluación de los beneficios sociales.

Con la aparición de la planificación de la familia, junto con la educación y otras esferas de inversión social como proyectos admisibles para los bancos, y especialmente con la decisión del Banco Internacional de Reconstrucción y Fomento y sus filiales, de financiar estas esferas de desarrollo, el análisis de costo/beneficio ha adquirido mayor importancia. Sin embargo, es necesario concentrar la mayor atención en el sector de los costos, ya que los beneficios han de ser de naturaleza controversial en el campo de la población. Importa explorar la eficacia de los costos de las distintas maneras de llevar a cabo los proyectos de población, incluso los programas de planificación de la familia. En la medida en que estos proyectos requieren una fuerza de trabajo relativamente escasa, importa lograr que se lleven a cabo de la manera menos costosa. No obstante, los análisis de costo/beneficio no necesariamente influirán sobre el proceso de decidir políticas, por cuanto otras consideraciones pueden inducir a los gobiernos a adoptar políticas de vital interés nacional. Los efectos no económicos, valorados por sus aspectos humanitarios y sociales, también motivan a quienes deciden las políticas, a pesar de que sus benéficas consecuencias no. pueden ser evaluadas en términos económicos.

Población óptima. El Comité reconoció que, además de las variables demográficas, el concepto de población óptima implicaba muchos criterios sociales y económicos como también apreciaciones de valor. En algunos análisis se consideró que un concepto óptimo dinámico reflejaría el cambio en las relaciones entre la población y los recursos provocado por el crecimiento de la población y el progreso tecnológico. Era difícil establecer empíricamente tal óptimo dinámico en lo referente al tamaño de la población y el Comité no lo consideró como una herramienta analítica útil para los fines de la comparación internacional. En cuanto a la tasa de crecimiento de la población, la tasa de fecundidad y la distribución de la población dentro del territorio nacional, pareció pertinente la optimación con respecto a las variables demográficas, especialmente con el propósito de formular políticas nacionales. Sin embargo, el Comité no hizo ninguna recomendación concerniente al uso internacional ya sea del crecimiento óptimo de la población o los criterios óptimos de distribución.

\section{PRoyecciones de POBLACtón}

Durante los últimos 20 años las Naciones Unidas han preparado proyecciones de la población total por edad y por sexo para el mundo, regiones y países. Estas proyecciones fueron utilizadas ampliamente para establecer 
cálculos de la población futura prevista en todas las esferas de la planificación a nivel nacional e internacional.

De esta manera, para los propósitos del Segundo Decenio de las Naciones Unidas para el Desarrollo, la División de Población preparó en 1968 proyecciones revisadas de la población total por edad y por sexa para todos los países y regiones. Se ha abarcado el período 1965-1985, proporcionando cuatro variantes para los países menos desarrollados, con mayor detalle en la estructura por edades que lo que ha sido posible hasta ahora. Estas proyecciones han proporcionado la base para que los miembros competentes del sistema de las Naciones Unidas preparen proyecciones de la población económicamente activa (sector de la oferta), la población urbana y rural, la población agrícola y no agrícola, la asistencia a la escuela y los hogares y las familias. Todas estas proyecciones están estandarizadas y coordinadas en el sentido de que usan una clasificación uniforme de los países y las regiones, tienen las mismas categorías básicas de edades y sexos y se refieren a períodos de tiempo similares.

Una vez preparadas, estas proyecciones abarcarán el período de 1950 a 1985 haciendo posible una variedad de estudios : a) análisis representativos de variables económicas y demográficas; $b$ ) estudio de las correlaciones entre variables económicas y demográficas básicas con referencia especial al examen de las fluctuaciones en las variables de población en diferentes situaciones de desarrollo; $c$ ) simulaciones o proyecciones, o ambas, en el futuro más distante.

Todavía se necesita una mayor coordinación de los supuestos en este conjunto de proyecciones demográficas que están preparando las Naciones Unidas y sus organismos especializados. Es importante tomar en cuenta, por ejemplo, las relaciones entre las poblaciones urbana y rural, por una parte, y las poblaciones agrícolas y no agrícolas, por otra; entre la asistencia a la escuela y la participación en la fuerza de trabajo; entre la urbanización y la participación en la fuerza de trabajo y la fecundidad, etc. Son esferas importantes la producción y el consumo de alimentos, la industrialización, los cambios en la demanda de la fuerza de trabajo, las ganancias en el ingreso por habitante, las calificaciones de la fuerza de trabajo, el tamaño de las familias y la descendencia final.

La División de Población ha preparado también proyecciones de la población total por edad y por sexo para las diferentes regiones del mundo hasta el año 2000 donde se indica que los países menos desarrollados pueden tener a fines del siglo una población de 5040 millones, o sea 2790 millones más que en 1970. Estos cálculos se basan en la suposición de que tanto el crecimiento económico como los programas nacionales de planificación de las familias estimularán una reducción muy significativa en la tasa de reproducción bruta. Las mismas proyecciones indican también que los países menos desarrollados pueden tener dentro de los próximos 30 años incrementos de alrededor del $100 \%$ de la población en edad escolar, $145 \%$ de la población en edad de trabajo y $140 \%$ de las mujeres en edad de procrear.

La importancia general de la labor en el campo de las proyecciones hace urgente mejorar las actuales herramientas técnicas de proyección. Deben revisarse los modelos de tablas de mortalidad existentes y elaborarse medios para calcular las declinaciones futuras en la mortalidad como función del progreso médico, la salud pública y el mejoramiento en la nutrición y los niveles de vida. Deben mejorarse los métodos actuales para proyectar la fecundidad por edades, con objeto de tomar en cuenta los cambios en la edad para el matrimonio y en el espaciamiento de los nacimientos. Los métodos actuales para proyectar la migración son inadecuados; ninguno puede llevarse a la práctica en forma satisfactoria sin evaluar los factores sociales y económicos. 
Finalmente, debe insistirse en que las repercusiones de las tendencias de población previstas sobre la elaboración de políticas y la planificación deben ser estudiadas cuidadosamente tanto por los investigadores como por quienes deciden las políticas. Hay problemas vitales en la forma de satisfacer las diversas necesidades de la población que crece y se desarrolla, lo que incluye la alimentación, la vivienda, la educación, la sanidad y el empleo. También es necesario resolver los urgentes problemas de la superpoblación agrícola, la industrialización, el uso del espacio urbano y el deterioro del medio ambiente que se atribuyen, en parte, a los desequilibrios entre la población, los recursos y las instituciones.

El Comité opinó que debía mantenerse y ampliarse la labor de proyección de la División de Población para que reflejara la necesidad cada vez mayor de datos más detallados sobre el cambiante carácter económico y social y la composición demográfica de la población mundial. Con el objeto de formarse una idea acerca de los posibles acontecimientos demográficos futuros, el Comité consideró la conveniencia de extender las proyecciones de población más allá del año 2000. Si bien esas proyecciones son por naturaleza especulativas e hipotéticas, esos ejercicios de simulación pueden resultar esclarecedores. Se hizo la distinción, sin embargo, entre las proyecciones de mediano plazo para varios decenios basadas en pruebas empíricas y un consenso de apreciaciones y las proyecciones de población a muy largo plazo, digamos, más allá del año 2000, que las Naciones Unidas debían considerar con mayor renuencia. Las incertidumbres que van ligadas a estos ejercicios demográficos a largo plazo limitan su utilidad para los propósitos de la planificación, que raramente va más allá de uno o dos decenios. E1 Comité, por consiguiente, recomendó que se concentrara la labor en las proyecciones detalladas de mediano alcance pero que debía continuar también el trabajo exploratorio del período posterior al año 2000, tomándose en cuenta una amplia serie de opciones con propósitos ilustrativos.

\section{CONSEcuencias para las políticas}

En cualquier discusión de las relaciones recíprocas entre los factores demográficos y económicos del desarrollo, resulta evidente que en casi todas las etapas de la discusión se plantean cuestiones importantes sobre política. Difícilmente se halla un gobierno que, al poner en práctica un programa de desarrollo, no afecte a factores de la vida social y económica que influyen sobre las principales variables económicas, el empleo, los ingresos, la tenencia de la tierra, el nivel de la capacidad, el grado de instrucción, la disponibilidad de viviendas, etc. y que por su conducto no afecte la dimensión de la familia, la mortalidad, la migración, y además, la edad y la estructura social y profesional de la población. Este factor no ha sido siempre reconocido de modo explícito, aunque se reconocen cada vez más estas relaciones en la mayoría de los países, sobre todo entre los países en desarrollo, sea cual fuere la tasa de desarrollo, el nivel de los ingresos, la tasa de crecimiento demográfico y el volumen existente de la población, y aun en el caso de distintos sistemas económicos y sociales. A su vez, en algunos casos existe una política encaminada al desarrollo de la población, lo cual comprende su crecimiento, estructura y distribución, ya sea de modo explícito o implícito, que puede tener repercusiones en la política del desarrollo que no siempre se perciben con claridad. En términos generales, el aumento en la tasa de crecimiento de la población de muchos países en desarrollo ha sido tan brusco que los gobiernos no han podido elaborar políticas de desarrollo que tomen debidamente en cuenta las consecuencias inmediatas y previsibles de dicho crecimiento. Los problemas del desarrollo 
social y económico derivados de la rigidez en la estructura social y las imperfecciones en la estructura internacional, así como de la falta de una política adecuada de desarrollo, se complican aún más por las tasas elevadas de crecimiento de la población.

Durante un período futuro bastante largo, será preciso dar por sentadas las tendencias en la dimensión absoluta de la población cuyas necesidades en la esfera económica y del bienestar social deben ser satisfechas. Es indis. pensable que los esfuerzos productivos se encaminen a mejorar constantemente el nivel de vida, las realizaciones sociales y culturales, así como el bienestar, con objeto de conseguir que los amplios sectores de la población con nivel de vida más bajo reciban los beneficios de la mayor productividad. Evidentemente, ello tiene consecuencias internacionales. Dadas las distintas tasas de crecimiento económico de la población en diferentes partes del mundo, hay sin embargo una interdependencia general que debería reflejarse en las políticas tanto en el nivel internacional como en el nacional. Es perfectamente claro que esas políticas distan de ser consecuentes o armoniosas. Las políticas nacionales parecen perseguir en su mayor parte unos objetivos nacionales estrictamente definidos que no toman debidamente en cuenta las consecuencias internacionales.

Cuando el tema de la discusión se refiere a los componentes de la población y de la economía, es decir, el cambio estructural y sectorial, se hace evidente cierto número de relaciones concretas entre el crecimiento de la población y el crecimiento económico, que requieren un examen cuidadoso. La investigación empírica sobre la mayoría de estos temas concretos aún se halla en sus primeras etapas, aunque frecuentemente se han hecho formulaciones teóricas que parecen ganar creciente reconocimiento. Sin embargo, a menudo se elabora una política sin investigación previa e incluso sin un conocimiento adecuado de los hechos. La meta sería estudiar todas las consecuencias de las políticas sociales y económicas con objeto de comprender sus repercusiones $\mathrm{y}$, cuando así proceda, revisarlas y ajustarlas a los propósitos generales.

Aun si se lograran grandes progresos en la reducción de la fecundidad, el hecho inevitable es que los países en desarrollo tendrán que doblar al menos la escala de sus instalaciones sociales y productivas y de sus servicios públicos con el solo fin de atender el aumento previsto de la población en los próximos 30 años. Tal es la razón fundamental de que la explosión demográfica sea un problema formidable para las aspiraciones de elevar el nivel de vida y mejorar la calidad de la vida humana.

E1 aspecto más general de este problema se relaciona con la formación de capital y la asignación de las inversiones. El crecimiento de la población no sólo aumenta la demanda de capital, sino que probablemente reduce también la capacidad de ahorrar. Los servicios sanitarios y educativos rinden sus beneficios lentamente, pero sólo si son compatibles con las inversiones directamente productivas, pero las grandes inversiones del tipo de las primeras, dedicadas a la población, aunque se necesitan para el desarrollo, reducen la capacidad para hacer inversiones de productividad más inmediata y con la facultad de crear empleos.

En la agricultura se suscitan problemas especiales. Los recientes descubrimientos en la tecnología agrícola han reducido la preocupación sobre el suministro adecuado de alimentos en un futuro inmediato. Sin embargo, es tal la rapidez del crecimiento de la población, que en muchos países el sector agrícola tendrá que expandirse rápidamente a fin de satisfacer las necesidades alimentarias y crear las oportunidades de empleo que se precisan. En ausencia de un desarrollo dinámico, existe el peligro claro y real de que se produzca una degeneración acumulativa de la situación rural. La rigidez de las estructuras agrícolas, tales como las vinculadas con la tenencia de la tierra y las relaciones con la mano de cbra, siempre fue 
un obstáculo mayor que se opuso al cambio económico y que exige reformas enérgicas. En muchos países dichas reformas parecen ser indispensables para evitar el empeoramiento de sus dificultades, que ya son agudas.

Al mismo tiempo debe ampliarse la capacidad de absorción de los sectores no agrícolas y de las regiones urbanas, a fin de rectificar los desequilibrios existentes en la oferta y la demanda, y a fin de aliviar las enormes presiones que se prevén para los próximos decenios. Asimismo, los sectores no agrícolas deberían proporcionar amplias oportunidades de empleo y elevar los niveles de los ingresos personales, actualmente inadecuados. En última instancia, la expansión de los sectores no agrícolas y urbanos es el mejor camino hacia el adelanto económico y en definitiva influye decisivamente sobre las modalidades de la procreación.

En la actualidad es evidente que ni el sector agrícola ni el urbano tienen la debida flexibilidad para ofrecer nuevas oportunidades de empleo al ritmo necesario. Se precisa una política más vigorosa de crecimiento y ampliación de las inversiones para remediar este defecto central. Ello requiere una administración dinámica, la selección de tecnologías apropiadas, y la capacitación de las categorías adecuadas de mano de obra calificada.

Los sistemas educativos deben ampliarse y transformarse con nuevas tecnologías a fin de poder resolver el problema actual. De modo semejante, los servicios de sanidad deben adaptarse a las exigencias de nuevas situaciones para las cuales no bastarán los conceptos tradicionales. El progreso tecnológico en la esfera productiva es de importancia esencial. Es indudable que la creatividad tecnológica podría resolver muchos de estos problemas si el dinamismo de la innovación apuntara más a resolver los problemas de los países en desarrollo.

Algunas de las medidas relacionadas con una política de población no darán resultado hasta dentro de algunos decenios, pero otras repercuten inmediatamente sobre una parte al menos de los pobladores. Ante todo, muchas políticas de población afectan al mismo tiempo los intereses de los padres, los hijos y los trabajadores. Existe un proceso acumulativo en las políticas de asistencia social. El mejoramiento en la salud y en otras esferas se refleja en una productividad en aumento así como en las condiciones generales de la vida.

En algunos países con grandes reservas no aprovechadas de recursos naturales o con territorios donde viven pocos habitantes, se advierte la inclinación comprensible a considerar las tendencias demográficas corrientes sobre la base de los problemas y perspectivas para el asentamiento de dichos territorios y la ampliación del mercado interno. En tales casos, puede suceder que a los gobiernos les preocupe mantener o incluso aumentar la tasa de crecimiento de la población y, al mismo tiempo, alcanzar un gran volumen de migración interna o atraer a una migración internacional. Un criterio acertado debería distinguir entre la necesidad a corto plazo de población en edad de trabajar y con la preparación necesaria, y las tendencias a largo plazo que provoca una tasa elevada de crecimiento de la población con sus problemas consiguientes, derivados del número creciente de personas jóvenes y no calificadas que tratan de entrar en una fuerza de trabajo urbana donde ya se registra exceso de oferta. Como parte del abjetivo general de alentar el desarrollo social y económico, indudablemente dichos países en el futuro querrán estudiar más a fondo que hasta ahora estas relaciones recíprocas.

Es obvio que uno de los medios más eficaces para mejorar el equilibrio entre la población, los recursos y el empleo consiste en liberalizar las condiciones de las corrientes de migración internacional, de comercio y de capital. En los decenios venideros la cooperación internacional se convertirá en una necesidad cada vez más imperiosa. El desarrollo autosuficiente necesitará particularmente la expansión de las oportunidades del comercio 
exterior, incluso la eliminación de las restricciones al comercio de productos de los países en desarrollo, la adopción de medidas para lograr precios estables y el mejoramiento de las condiciones de la ayuda externa, así como la ampliación de la cooperación técnica internacional. En cuestiones de política de población en relación con el desarrollo surgen necesidades especiales de cooperación internacional, como se menciona en otra parte de este informe.

En sus actividades de planificación, los gobiernos deberían reconocer que las asignaciones para inversiones regionales y sectoriales pueden influir profundamente sobre la fecundidad. La ubicación de la actividad industrial contribuye a una rápida modificación de las tasas de actividad, de la condición jurídica y social de la mujer, y de las normas sobre dimensión de la familia. Ninguna política de inversión tiene consecuencias neutrales respecto de la población, y entre las posibles políticas elegibles de esta índole debería darse prioridad sistemática a las que contribuyen al proceso de modernización general.

En el curso del proceso de modernización se alterarán las funciones económicas de la familia. Los gobiernos que reconocen este hecho tienen muchas oportunidades para acelerar el proceso al estimular a las instituciones que complementan las funciones tradicionales de la familia, ofreciendo seguridad para la vejez y protección en caso de enfermedad sin tener necesariamente que confiar en una familia numerosa.

Las políticas se establecen en forma positiva o por omisión. Sin embargo, la ausencia de una política de población es una política determinada por otros criterios. En cambio, una política de desarrollo económico discordante puede tener consecuencias sobre los cambios de la población. El Comité hizo un llamamiento a fin de que se formulasen de modo cada vez más explícito políticas consistentes, en conformidad con las aspiraciones nacionales y en armonía con los intereses a largo plazo de la humanidad en la esfera internacional. Fue también un llamamiento en favor de una investigación adecuada que ayude a elaborar y aplicar las políticas necesarias en el nivel internacional y nacional.

\section{INVESTIGACIONES Y MEDIDAS RECOMENDADAS}

Teniendo presente la resolución 1483 (XLVIII) del Consejo Económico y Social, relativa a la necesidad de realizar estudios en la esfera de la población durante el Segundo Decenio para el Desarrollo, el Comité convino en declarar en primer término lo que a su juicio eran amplias esferas de labor donde se necesitaban nuevas investigaciones, para prestar luego atención al papel del sistema de organizaciones de las Naciones Unidas en la realización de los estudios recomendados. Al formular sus recomendaciones, el Comité tuvo presente también las recomendaciones del Comité Especial de Expertos encargado de la preparación de un programa a largo plazo en ma. teria de demografía (E/CN.9/182 $)^{3}$ así como las del Seminario interregional

3 El Comité Especial recomendó cinco temas generales de investigación:

1) El estudio de la población como fuerza productiva, incluso la utilización de la mano de obra, el coeficiente de población activa por sexos y edad, ocupación e industria; la distribución por características sociales; y las correlaciones de estas factores con el crecimiento de la población, la migración interna, la urbanización y otros factores económicos y sociales;

2) El estudio de la población como conjunto de consumidores, incluso la estructura por edades y por sexos, las tasas del crecimiento de la población rural y urbana y de los diversos grupos profesionales, en relación con las necesidades de alimentos, vivienda, servicios de varios tipos y las correlaciones con la salud y la mortalidad, el matrimonio y la fecundidad y el tamaño de la familia; 
sobre la aplicación de los datos y estudios demográficos a la planificación del desarrollo celebrado en Kiev del 15 al 25 de septiembre de 1969 (E/CN. 9/223).4

Esferas donde se presentan problemas. La relación mutua entre el cre. cimiento demográfico y el crecimiento económico todavía no se comprende totalmente. Aunque el Comité reconoció que a los fines de la planificación y la formulación de políticas en el plano nacional se necesitan urgentemente macromodelos de los cambios económicos y demográficos, deberá emprenderse mucha investigación básica antes de que siquiera las interacciones más importantes entre el desarrollo demográfico y el económico se comprendan lo suficientemente bien como para optimar las políticas en el plano nacional.

En consecuencia, el Comité enumeró cierto número de esferas determinadas de interacción que necesitan urgentemente ser investigadas y analizadas :

1) ¿Cómo pueden las políticas de desarrollo y de población contribuir al rápido adelanto económico y social de la población?

2) ¿Cuál es la constelación de fuerzas económicas y sociales que afectan la fecundidad?

3) ¿Cómo influyen los niveles de fecundidad sobre la asignación de recursos familiares con respecto a la inversión y al ahorro, a la formación de capital humano y a las modalidades de consumo?

4) ¿Cómo influyen los cambios en la tasa de crecimiento de la población sobre otros ahorros, y especialmente sobre la asignación de recursos del sector público entre las diversas inversiones sociales y las "directamente productivas"?

5) ¿Cómo pueden las políticas de desarrolio económico mitigar mejor las posibles tendencias del rápido crecimiento de la población a generar desempleo?

6) ¿Cómo se adapta la tecnología al rápido crecimiento de la población, y qué cambios tecnológicos inducidos se requieren en vista del crecimiento de la población y de las proporciones y precios de los factores en los países en desarrollo?

3) El estudio de las características demográficas de los grupos por categoría social dentro de la población y de la relación mutua entre esos grupos y los factares económicos y la seguridad económica de las familias;

4) El estudio de las relaciones mutuas entre el desarrollo de la población, las tasas de crecimiento económico, la productividad económica, las necesidades de suministro de alimentos y de capital, incluso la magnitud de las correlaciones actuales y los factores conexos y las proyecciones con diversas hipótesis en cuanto a las tendencias futuras;

5) El análisis de la probable población futura según distintas hipótesis en cuanto a las relaciones mutuas entre los distintos factores del desarrollo económico, la mortalidad, la fecundidad y la migración.

4 El informe del Seminario contenía recomendaciones sobre los siguientes temas:

Estudios de población para la planificación del desarrollo;

Estudios sobre la mano de obra para la planificación del desarrolo con referencia a la industrialización;

Población, alimentación y agricultura;

Tendencias de la población y nivel de vida;

Características educacionales;

Análisis necesarios para la planificación en materia de sanidad;

Población, vivienda y hogares:

Migración interna y urbanización;

Desarrollo social;

Política demográfica: fecundidad y planificación familiar. 
7) ¿Cuál es el efecto del crecimiento de la población sobre la oferta y la redistribución interna de la mano de obra, y sobre el empleo tanto en los sectores urbanos como los rurales, y sobre la transformación estructural de la economía?

8) ¿Cómo decidirá un país sobre una política de desarrollo regional dinámica y óptima, y cómo la aplicará, y cuál es el papel de la densidad de la población comparada con otros factores de dicho desarrollo?

9) ¿Cómo afecta el proceso de desarrollo y urbanización a la condición jurídica y social y al papel económico de la mujer, y cuáles son los efectos de tales cambios sobre la edad para el matrimonio y la fecundidad?

10) ¿Cuáles son los efectos secundarios del desarrollo y la política en materia de población en las esferas económica, demográfica, social, étnica y genética?

11) ¿Qué cambios podrían hacerse en el comercio internacional y las políticas de asistencia en respuesta a las tendencias demográficas de los países en desarrollo?

Programa de investigaciones. Al formular y organizar programas en la esfera del desarrollo económico es indispensable tener en cuenta los aspectos demográficos del problema, y el Comité expresó la esperanza de que las Naciones Unidas, sus organismos especializados y las comisiones económicas regionales continuaran $\mathrm{y}$, cuando fuese necesario, intensificaran, la atención que conceden a los factores demográficos en sus programas de investigación en general.

Era muy importante que se conociera la información demográfica y económica pertinente sobre las dimensiones y el carácter de la población. El Comité recomendó que las Naciones Unidas continuaran su trabajo de uniformar y coordinar la reunión y difusión de datos demográficos, económicos y sociales. El Comité tomó nota al respecto del trabajo de la Comisión de Estadística de la Oficina de Estadística sobre los sistemas integrados de estadísticas sociales y demográficas con el sistema de cuentas nacionales ( $\mathrm{SCN}$ ) y el sistema de balances del producto material (SPM).

Una recomendación a la cual el Comité concede prioridad es la de que las Naciones Unidas continúen la labor de comparar y analizar datos demográficos y económicos. El Comité estimó que sería conveniente iniciar, sobre la base de los datos del período de posguerra, un número creciente de estudios de sectores representativos y de series cronológicas del desarrollo económico y el cambio demográfico, estableciendo las correlaciones y los factores conexos y preparando estimaciones respecto de las tendencias y los acontecimientos futuros.

El Comité recalcó, en relación con la importancia de disponer de información adecuada sobre crecimiento de la población para las políticas y la planificación del desarrollo y con respecto a nuevos estudios de las relaciones mutuas económico-demográficas, la necesidad de continuar y extender la labor sobre las proyecciones demográficas amplias emprendidas por el sistema de las Naciones Unidas.

Los apremiantes problemas del empleo que pueden crearse por razón del rápido crecimiento de la población llevaron al Comité a recomendar que se realizaran nuevas investigaciones para aislar los determinantes de las tasas de participación en la mano de obra por sexo y por edad, como ayuda para estimar las tendencias de la mano de obra en los diferentes países; que se prepararan proyecciones de la fuerza de trabajo para ser coordinadas con las políticas y planes gubernamentales e integradas en ellos; que se estudiaran las consecuencias de un rápido crecimiento de la población y de la mano de obra sobre la tecnología que crea empleos, y sobre la capacidad de los programas de desarrollo de absorber no sólo a los nuevos trabaja. 
dores que ingresan en el sector laboral, sino también los que tienen empleo insuficiente o están desempleados.

Los problemas de la migración interna, de la movilidad entre sectores y del cambio estructural, en opinión del Comité, requieren investigación a fondo con objeto de determinar sus causas y efectos sobre las perspectivas del desarrollo. Para efectuar dicha investigación se necesitan datos mejores e internacionalmente más comparables sobre las corrientes de migración. Para comprender mejor el proceso de desarrollo y poder formular las políticas y los programas adecuados, se requería tener un conocimiento más adecuado de las relaciones mutuas entre los cambios estructurales de la población y la economía, con sus repercusiones sobre los niveles de vida, el empleo, la fecundidad y la mortalidad.

El Comité sugirió que, habida cuenta de la continua importancia de población agrícola en los países en desarrollo, debía prestarse especial atención a las relaciones mutuas entre la población y los recursos naturales y agrícolas disponibles, prestando debido reconocimiento al efecto de las nuevas tecnologías que surgen en esta esfera.

El Comité, al observar que las consecuencias acumulativas del rápido crecimiento de la población y de la alta fecundidad sobre la distribución del ingreso personal y sus consecuencias sobre el creciente desempleo y sobre las poblaciones marginales, se comprendían sólo en forma vaga, insistió en la necesidad de emprender investigaciones en esta esfera en los planos macro y microeconómicos.

Un objetivo fundamental de investigación subrayado reiteradamente por el Comité es la necesidad de entender mejor y predecir más exactamente las variaciones en el comportamiento de la fecundidad. Se creyó que el análisis del comportamiento económico y demográfico de la familia era una nueva orientación provechosa para la investigación teórica y empírica destinada a mejorar las técnicas para predecir los cambios demográficos. Se pensó que esas técnicas analíticas eran necesarias para evaluar la eficacia de las políticas que tienen influencia explícita o implícita sobre la fecundidad y el crecimiento de la población.

El Comité expresó reservas respecto de lo adecuado de las metodologías corrientes que tratan de evaluar los beneficios derivados de los cambios demográficos, tales como programas de planificación de la familia. No obstante, el claro valor social, económico y humanitario de los programas de salud pública y de planificación de la familia debía estudiarse a fondo con el fin de evaluar su eficacia. Debía apoyarse la realización de estudios de costo/eficacia en todas las esferas de los gastos sociales a fin de respaldar sus pedidos de mayor participación en los escasos recursos públicos.

El Comité observó la tendencia hacia una mayor aplicación de las téc. nicas de análisis del costo/beneficio a los programas de desarrollo. Sin dejar de reconocer las limitaciones que entraña la medición de los beneficios, el Comité estimó que debía alentarse esa tendencia. Instó a que el sistema de las Naciones Unidas considerara la utilidad de estas técnicas de evaluación de políticas en la esfera de la educación, la salud y la planificación de la familia, entre otras, y en conexión con ello, reuniera datos de diferentes países con objeto de facilitar las comparaciones internacionales en esas esferas.

Los miembros del Comité sugirieron que debía dedicarse más atención a la tarea de determinar la contribución de la nutrición, la salud pública y de los niveles de vida al desarrollo mental y físico de la población infantil del mundo, y a estudiar las causas determinantes de la mortalidad y morbilidad de los niños y los lactantes, así como las consecuencias que para la productividad tienen las mejoras en la esfera sanitaria. El Comité creyó que ese tipo de investigación podría documentar la importante contribución 
aportada por los programas de higiene maternoinfantil y de planificación de la familia al bienestar social y económico de las poblaciones.

Función del sistema de las Naciones Unidas. Luego de haber examinado los distintos aspectos demográficos del desarrollo económico y formulado recomendaciones respecto de programas y prioridades en esta esfera, el Comité dirigió su atención a la función del sistema de organizaciones de las Naciones Unidas en la realización y promoción de las investigaciones recomendadas.

El Comité convino en que la población era una de las esferas más importantes para la cooperación internacional en el Segundo Decenio para el Desarrollo, cuya estrategia fue el tema de dos Comisiones de la Asamblea General durante los dos últimos años. Los miembros oyeron también que, en este aspecto, el Consejo Económico y Social, por su resolución 1483 (XLVIII), había pedido al Secretario General que "en consulta con los organismos competentes, continúe estudiando las relaciones que existen entre el crecimiento demográfico y el desarrollo económico y social, teniendo especialmente en cuenta el aumento del producto nacional bruto y la eleva. ción del ingreso nacional per capita y el nivel de vida, y difunda ampliamente los resultados principales de esos estudios".

EI Comité advirtió perfectamente las consecuencias de dicha resolución sobre los programas en los aspectos demográficos del desarrollo económico que había recomendado. Se señaló que la materia era casi totalmente nueva en lo relativo a la participación de las Naciones Unidas y los miembros, por ello, destacaron la importancia de movilizar todos los recursos disponibles a fin de llevar a cabo los proyectos de investigación recomendados, no solamente en los planos internacional y regional, sino también en el nacional.

El Comité oyó declaraciones relativas a la competencia y conocimientos técnicos especiales de las Naciones Unidas, la OMS, la OIT y la UNESCO en la esfera de la población, así como en lo relativo al correspondiente programa de trabajo de la CEPALO. Se informó al Comité del mecanismo de "coordinación" que existía en el sistema de las Naciones Unidas, es decir el Comité Administrativo de Coordinación y su Subcomité de población.

Aunque la mayor parte de las esferas y temas de investigación que trató el Comité estaban comprendidos, al menos en parte, dentro de la competencia del sistema de las Naciones Unidas, el Comité estimó que debían realizarse esfuerzos con la cooperación de la asistencia técnica, e incluso del Fondo de las Naciones Unidas para Actividades en Materia de Población, a fin de alentar y apoyar la investigación en el plano nacional. El Comité opinó que el sistema de las Naciones Unidas debía proporcionar asistencia técnica y financiera a los países miembros para ayudarles a mejorar su base de datos demográficos y económicos para la investigación, planificación y adopción de políticas. El Comité insistió sobre la importancia de que se aliasen los institutos de investigación gubernamentales y no gubernamentales en los países miembros, e instó a que concentraran sus investigaciones en el estudio de las relaciones mutuas entre el desarrollo econó mico y el demográfico. El sistema de las Naciones Unidas debía fomentar, mediante asistencia técnica y financiera, la capacidad de investigación en demografía y economía, y promover intercambios internacionales de los resultados de las investigaciones con el fin de acelerar la difusión de los conocimientos en esta esfera. El Comité también creyó que el sistema de las Naciones Unidas estaba particularmente capacitado para promover las comunicaciones en la esfera de la investigación demográfica y económica relacionada con la formación de políticas y la planificación.

Al respecto, el Comité tomó nota de la cooperación técnica de que podía disponerse por conducto del sistema de las Naciones Unidas en muchas 
esferas, entre ellas capacitación, investigación, servicios de información y de asesoramiento, así como en los mejores métodos para aplicar los programas. 5 El Comité encomió esta formulación de programas de cooperación técnica y expresó la esperanza de que se recibiesen recursos de dinero y personal suficientes para continuar la recomendada expansión en las esferas de interés para el Comité, en conformidad con la resolución 1483 (XLVIII) del Consejo de Seguridad.

5 Dichos métodos son: a) mayor asistencia para la elaboración de medidas económicas, sociales y de otro tipo en materia de población, incluido el desarrollo de la planificación de la familia; $b$ ) mayor asistencia para el desarrollo de programas y proyectos nacionales, sobre todo los proyectos que tienden a la determinación de políticas, incluido el desarrollo de los servicios de investigación demográfica que se precisan para la formulación de políticas; $c$ ) iniciación de una serie de proyectos bien equilibrados y autónomos en los planos nacional y regional; d) mayor insistencia en la capacitación del personal nacional necesario para encargarse de los programas nacionales en materia de población y otorgamiento de las becas necesarias; $e$ ) reforzamiento de la infraestructura de las Naciones Unidas para poder hacer frente a las exigencias de un programa de cooperación técnica en expansión. (Véase E/4768, párr. 140.) 